\title{
NUEVOS ORIBÁTIDOS (ACARIFORMES, ORIBATIDA) PARA LA FAUNA DE LA PENÍNSULA IBÉRICA
}

\author{
L. S. Subías $(*)$
}

\begin{abstract}
RESUMEN
Como resultado del estudio de nuevas muestras de suelo procedentes de diversas localidades de la Península Ibérica, se ha realizado el presente trabajo en el que se citan por primera vez en el área de estudio una familia, cuatro géneros, 19 especies y dos subespecies; se dan dos nuevos nombres, Chamobates confusus y Oribatula tibialis allifera, y se crea un nuevo subgénero, Minunthozetes (Inigozetes), al mismo tiempo que se hacen tres nuevas combinaciones genéricas y una nueva sinonimia.

Palabras clave: Faunística, Taxonomía, Península Ibérica, Nuevas citas, Chamobates confusus n. nom., Oribatula tibialis allifera n. nom., Minunthozetes (Inigozetes) n. subgen.
\end{abstract}

\begin{abstract}
New oribatid mites (Acariformes, Oribatida) for the Iberian Peninsula fauna

As result of the study of recent soil samples from various sites of the Iberian Peninsula, one family, four genera, 19 species and two subspecies are recorded for the first time for the study area; two new names, Chamobates confusus and Oribatula tibialis allifera, are given, and a new subgenus, Minunthozetes (Inigozetes), is erected; also three new generic combinations and a new sinonimie are maked.

Key words: Faunistic, Taxonomy, Iberian Peninsula, New records, Chamobates confusus n. nom., Oribatula tibialis allifera n. nom., Minunthozetes (Inigozetes) n. subgen.
\end{abstract}

\section{Introducción}

A pesar de que la Península Ibérica muestra una gran variedad de condiciones ambientales y una enorme biodiversidad, el conocimiento de su fauna de ácaros oribátidos es uno de los mejores de todo el mundo, elevándose el censo de las especies y subespecies válidas actualmente concidas a 772 793 si consideramos las nuevas citas del presente trabajo- - lo que supone más de un $10 \%$ de la fauna mundial hasta ahora conocida. A pesar de ello, todavía son muchas las nuevas especies, tanto para la ciencia como para la fauna peninsular, que continuamente siguen apareciendo, siendo estas últimas el objeto fundamental del presente trabajo ya que, como consecuencia de las recientes prospecciones realizadas, sobre todo en medios estrictamente endógeos procedentes de taludes arenosos o suelos profundos - a veces en zonas extremadamente áridas- ha aparecido una fauna relicta de características tropicales muy emparentada con la etiópica actual.

* Departamento de Biología Animal I. Facultad de Biología. Universidad Complutense. 28040 Madrid. 


\section{Resultados}

Familia PARHYPOCHTHONIIDAE Grandjean, 1932

Parhypochthonius aphidinus Berlese, 1904

También el género Parhypochthonius Berlese, 1904 y la familia Parhypochthoniidae son citados por primera vez en la Península Ibérica. Su presencia era esperada ya que se trata de una especie comopolita (Subías y Gil-Martín, 1997) ya conocida del noroeste de Italia. Su localidad de recolección ha sido Llabería (Tarragona), en el noreste peninsular.

\section{Familia BRACHYCHTHONIIDAE Thor, 1934}

Sellnickochthonius jugatus (Jacot, 1938)

Aunque esta especie ya había sido citada por primera vez en España por Subías (1977), posteriormente — siguiendo el criterio de Moritz (1976) — se asignan dichos ejemplares, y los citados posteriormete por Subías (1980), a Sellnickochthonius suecicus (Forsslund, 1942). Más adelante, Balogh y Mahunka (1983) diferencian S. jugatus —a la que consideran de distribución holártica- de la cosmopolita $S$. suecicus porque en la primera de las especies las setas notogastrales centrales son algo engrosadas (pudiendo presentar también alguna barbulación), mientras que en la segunda dichas setas son setiformes y lisas. Volviendo a revisar el material español se ha podido comprobar que, al menos los ejemplares de la Sierra de Cazorla (Subías, 1980), presentan las setas notogastrales como el auténtico $S$. jugatus, motivo por el cual se vuelve a reinstaurar esta especie dentro de la fauna ibérica.

Familia COSMOCHTHONIIDAE Grandjean, 1947

Cosmochthonius maroccanus Gil-Martín, Subías y Arillo, 1992

Especie desrcrita recientemente del norte de África por Gil-Martín et al. (1992) y que ha vuelto a ser encontrada en el sur de Portugal, en Sines.

\section{Familia LOHMANNIIDAE Berlese, 1916}

Lohmannia loebli Mahunka, 1974

Especie descrita originalmente de Grecia y que Subías y Gil-Martín (1997) consideran de distribución mediterránea, por lo que tampoco era de extrañar su presencia en la Península Ibérica, donde ha sido encontrada en el centro, Ocaña (Toledo), y sureste, Gádor (Almería), así como también en las islas Baleares, Ciudadela (isla de Menorca).
Thamnacarus deserticola (Grandjean, 1934)

Especie descrita de Marruecos y de distribución actual paleártica meridional (Subías y Gil-Martín, 1997), por lo que su presencia en el sureste español, en el Cabo de Gata (Almería), era de esperar. El género Thamnacarus Grandjean, 1950, es la primera vez que se cita en la Península Ibérica.

\section{Familia PHTHIRACARIDAE Perty, 1841}

Phthiracarus serrulatus Parry, 1979

Especie probablemente euroatlántica ya que se la conocía de Gran Bretaña y Rumanía. La cita española corresponde a una zona montana de la provincia de Cuenca (Sierra de Mira).

Familia STEGANACARIDAE Niedbala, 1986

\section{Steganacarus (S.) doumandji Niedbala, 1986}

Especie hasta ahora sólamente conocida de su localidad típica, Argelia. La nueva cita procede del este de la Península Ibérica, de la sierra de Mira (Cuenca).

Steganacarus (Tropacarus) desmeti Niedbala, 1988

Especie descrita de Argelia y que no había vuelto a ser citada hasta ahora que ha sido encontrada en el centro de Portugal, en el Pico da Estrela.

Familia GYMNODAMAEIDAE Grandjean, 1954

Arthrodamaeus bicristatus L.S. Subías, Arillo y J. Subías, 1997

Especie descrita muy recientemente de Marruecos en una revisión del género Arthrodamaeus Grandjean, 1954, llevada a cabo por Subías et al. (1997). Por lo tanto, su presencia en el suereste español, en el cabo de Gata (Almeria), también era de esperar.

Familia MICROZETIDAE Grandjean, 1936

Miracarus senensis Bernini, 1975

Descrita del noroeste de Italia, esta especie no había vuelto a ser citada hasta ahora que ha aparecido en el levante español, en Pego (Alicante).

Familia DAMAEOLIDAE Grandjean, 1965

\section{Damaeolus asperatus (Berlese, 1904)}

Especie holártica que ya era conocida de diversas localidades del Mediterráneo occidental (sureste de Francia, oeste de Italia, Sicilia y Túnez), por lo que su presencia en la Península Ibérica era de 
esperar. La nueva cita procede del levante español, de Pego (Alicante).

\section{Familia AMEROBELBIDAE Grandjean, 1954}

Berndamerus bicostatus (Berlese, 1910)

El género Berndamerus Mahunka, 1977, presenta una distribución mediterránea y hasta ahora no había sido citado en la Península Ibérica. Esta rara especie, descrita del sur de Italia por Berlese (1910) y posteriormente encontrda en Grecia por Sellnick (1931), ha sido hallada ahora en el árido sureste español, en la Sierra de Gádor (Almería), procedente de una muestra endógea.

\section{Familia OPPIIDAE Grandjean, 1951}

Berniniella aeoliana (Bernini, 1973)

Especie considerada por Subías y Gil-Martín (1997) como mediterránea occidental, aunque recientemente Vasiliu et al. (1993) la han citado también de Rumanía. La nueva cita ibérica procede de Teixoso, en el centro de Portugal.

\section{Berniniella exempta (Mihelcic, 1958)}

Especie hasta ahora sólo conocida de Austria (de donde fue descrita) y del Cáucaso. La actual cita española procede de La Bonaigua, en el Pirineo leridano, aunque los ejemplares han sido adscritos a dicha especie provisionalmente ya que presentan algunas diferencias con la descripción original.

\section{Familia SUCTOBELBIDAE Jacot, 1938}

Suctobelbella italica (Mahunka, 1966)

Especie que desde su descripción de Italia por Mahunka (1966) no había vuelto a ser citada, habiendo sido recolectada en Pego (Alicante).

\section{Familia ACHIPTERIIDAE Thor, 1929}

Cerachipteria digita digita Grandjean, 1935

Subespecie de distribución mediterráneo occidental (Subías y Gil-Martín, 1997), ya citada de Andorra por Travè (1956). En España ya se conocía la subespecie Cerachipteria digita pyrenaica Pérez-İ̃nigo jr., 1991, pero se encuentra ahora por primera vez, tanto en España, puerto de La Bonaigua (Lérida), como en Portugal (Geres), ambas citas del norte peninsular.

Parachipteria patavina (Oudemans, 1913)

Consierada por Subías y Gil-Martín (1997) como mediterránea, no había sido citada hasta ahora en la Península Ibérica, aunque muy probablemente pertenezcan a dicha especie los ejemplares que Saloña e Iturrondobeitia (1988) citan como Parachipteria sp. y que proceden del norte de España, del País Vasco.

\section{Familia ORIBATELLIDAE Jacot,1925}

\section{Oribatella luisae Bernini, 1979}

Especie hasta ahora endémica del este del Mediterráneo occidental (Italia peninsular, Cerdeña y Sicilia). La nueva cita procede del noroeste español, de Boiro (La Coruña).

\section{Familia CERATOZETIDAE Jacot, 1925}

\section{Melanozetes meridianus Sellnick, 1928}

Especie holártica septentrional —o boreoalpina (Seniczak, 1989) - a la que probablemente pertenezcan varios ejemplares encontrados en el norte de España, en el puerto de Palombera (Cantabria).

\section{Familia CHAMOBATIDAE Thor, 1938}

\section{Chamobates confusus n. nom.}

(para Chamobates pusillus "sensu" Pérez-Iñingo, 1972, no Berlese, 1895)

Mahunka y Mahunka-Papp (1995), al redescribir los oribátidos de la "colección Berlese", consideran que el auténtico Chamobates pusillus (Berlese, 1895) se corresponde con lo que hasta ahora se venía denominando como Chamobates borealis (Trägardh, 1902), mientras que el C. pusillus "sensu" otros autores sería una especie diferente. Como existe una buena descripción de dicha especie realizada por Pérez-Íñigo (1972) —autor que además considera que existe más de una especie bajo dicha denominación-, se considera que es la más adecuada para que sirva de tipo para la nueva especie, a la que se da aquí el nombre de Chamobates confusus debido a la confusión existente ya mencionada.

\section{Familia MYCOBATIDAE Grandjean, 1954}

Jugatala cribelliger (Berlese, 1904) n. comb.

Considerada recientemente por Mahunka y Mahunka-Papp (1995) dentro del género Mycotabes Hull, 1916, tal vez sea más adecuado, por su tridactilia y porque no parece presentar los pteromorfos móviles, incluirla dentro del género Jugatala Ewing, 1913 — género que también se cita por primera vez en la Península Ibérica-. Esta especie sólo era conocida del norte de Italia y de los 
Alpes suizos. Las nuevas citas ibéricas proceden del centro de Portugal (Pico da Estrela) y del norte de España, de San Miguel de Aralar (Navarra), siendo muy probable que también deba de ser asignada a esta especie la cita que del País Vasco realizan Saloña e Iturrondobeitia (1988) bajo la denominación de Mycobates sp.

Minguezetes insignis (Berlese, 1910) n. comb.

Especie descrita de Sicilia como Punctoribates insignis y que muy posteriormente fue citada de Japón por Fujikawa (1981). Su presencia ahora en España, en la sierra de Mira (Cuenca), es la tercera cita de la misma, aunque muy probablemente la especie cosmopolita Minguezetes manzanoensis (Hammer, 1958) deba de ser considerada como nuevo sinónimo suyo a partir de la redescripción que de los ejemplares de $P$. insignis de la "colección Berlese" hacen Mahunka y Mahunka-Papp (1995), siendo entonces precisa su nueva ubicación dentro del género Minguezetes Subías, Kahwash y Ruiz, 1990.

\section{Minunthozetes (Inigozetes) n. subgen.}

En 1969, Pérez-Íñigo describe de España central la especie Minunthozetes reticulatus, que se diferencia de todas las demás del género por presentar sáculos notogastrales en lugar de áreas porosas. Como este carácter en todos los demás grupos de oribátidos poronóticos se utiliza habitualmente para la separación de géneros, es de pensar que, al menos, sirva para la división del género Minunthozetes Hull, 1916 en dos subgéneros, el típico, con áreas porosas y el nuevo, Minunthozetes (Inigozetes) — dedicado a la memoria del prestigioso oribatólogo español D. Carlos Pérez-Íñigo-, con sáculos.

ESPECIE TIPO: Minunthozetes reticulatus PérezÍñigo, 1969.

Familia GALUMNATIDAE Jacot, 1925

Allogalumna integer (Berlese, 1904)

Especie descrita del sur de Italia por Berlese (1904) y que no había vuelto a ser citada hasta ahora, habiendo aparecido en España central, en Guadalix (Madrid).

Pilogalumna crassiclava longiareata Pérez-Íñigo, 1976

Subespecie descrita de las islas Canarias, donde ha aparecido en diversas islas (Tenerife, Fuerteventura y Gran Canaria), pero fuera de las cuales no había sido encontrada hasta ahora. La nueva cita procede del centro de la Península Ibérica, también de Guadalix (Madrid).

Familia ORIBATULIDAE Thor, 1929

Oribatula tibialis allifera n. nom.

(para Oribatula tibialis alata Iordansky, 1991, "nom. praeoc." por Oribatula alata Mihelcic, 1956)

Descrita del Cáucaso por Iordansky (1991), esta subespecie también ha sido encontrada en la Península Ibérica, tanto en Portugal central como en el noreste y este de España, puerto de La Bonaigua (Lérida) y Sierra de Mira (Cuenca), respectivamente.

\section{Referencias}

Balogh, J. \& Mahunka, S., 1983. Primitive Oribatids of the Palaearctic Region. En: The Soil Mites of the World, 1. Elsevier, Amsterdam: 372 pp.

Berlese, A., 1904. Acari nuovi. Manipulus III. Redia, 2 (1905): 10-32.

Berlese, A., 1910. Acari nuovi. Manipuli V-VI. Redia, 6: 199-234.

FUJIKAWA, T., 1981. Oribatid fauna from nature farm in Nayoro (3). Edaphologia, 24: 19-24.

Gil-Martín, J., Subías, L.S. \& Arillo, A., 1992. Oribátidos de Marruecos y Sahara Occidental I: O. Inferiores (Acari, Oribatida, Macropylina). Graellsia, 48: 53-63.

IORDANSKY, S.N., 1991. Taxonomic revision of the Oribatid Mites Oribatula (Acariformes, Cryptostigmata, Oribatulidae) of the USSR Fauna. (En ruso). Zoologicheskii Zhurnal, 70: 77-89.

MahunKa, S., 1966. A study of Oribatids collected by Prof. Dr. Di Castri on the Mt. Spitz (Recoaro, Italy). Atti - Istituto veneto di scienze, lettere ed arti (Classe di scienze fisiche, matematiche e naturali), 124: 369-386.

MahunKa, S. \& MahunKa-Papp, L., 1995. The Oribatid species described by Berlese (Acari). Hungarian Natural History Museum, Budapest: 325 pp.

Moritz, M., 1976. Revision der europäischen Gattungen und Arten der Familie Brachychthoniidae (Acari, Oribatei). Teil 1. Mitteilungen aus dem Zoologischen Museum in Berlin, 52: 27-136.

PÉreZ-ÍÑIGO, C., 1969. Nuevos oribátidos de suelos españoles (Acari, Oribatei). Eos, 44 (1968): 377-403.

PÉrez-ÍÑIGO, C., 1972. Ácaros oribátidos de suelos de España Peninsular e Islas Baleares (Acari, Oribatei). Parte IV. Eos, 47 (1971): 247-333.

SAloña, M. \& Iturrondobeitia, J.C., 1988. Estudio de las comunidades de oribátidos (Acari, Oribatei) de varios ecosistemas de Bizkaia y una zona próxima: 1 . 
Sistemática y listado faunístico. Kobie (Serie Ciencias Naturales), 17: 79-92.

SEllnicK, M., 1931. Acari. En: M. Beier, Zoologische Forschungreise nach den Ionischen Inseln und dem Peloponnes, XVI. Sitzungsberichte der Akademie der Wissenschaften in Wien, 140: 693-776.

SENICZAK, S., 1989. The morphology of juvenile stages of moss-mites of the subfamily Sphaerozetinae (Acarida: Oribatida), I. Annales Zoologici, 42: 225-235.

SuBíAs, L.S., 1977. Taxonomía y ecología de los oribátidos saxícolas y arborícolas de la Sierra de Guadarrama (Acarida, Oribatida). Trabajos de la Cátedra de Artrópodos, Facultad de Biológicas, Universidad Complutense de Madrid, 24: 379 pp.

SubÍAs, L.S., 1980. Ácaros oribátidos de la Sierra de Cazorla (Acarida, Oribatei). En: M.G. de Viedma, Fauna de Cazorla. Invertebrados. I.C.O.N.A., Monogr. 23: 7-51.

Subías, L.S., Arillo, A. \& Gil-Martín, J., 1994. Oribátidos de Marruecos y Sahara Occidental, II. Listado de especies (Acari, Oribatida). Boletín de la Real Sociedad Española de Historia Natural (Sección Biológica), 91: 129-134.

Subías, L.S., Arillo, A \& Subías, J., 1997. The genus Arthrodamaeus Grandjean (Acariformes, Oribatida, Gymnodamaeidae). Acarologia, 38: 297-307.
Subías, L.S. \& Gil-Martín, J., 1997. Systematic and biogeographic checklist of Oribatids from Western Mediterranean (Acari, Oribatida). Annali del Museo Civico di Storia Naturale "G. Doria”, 91: 459-498.

Travé, J., 1956. Contribution à l'étude de la faune de la Massane (Deuxième note). Oribates (Acariens): 1ere partie. Vie et Milieu, 7: 77-94.

VAsiliu, N., IVAN, O. \& VASILIU, M., 1993. [The faunistic synopsis oribatids (Acarina: Oribatida) from Romania.] (En rumano). Anuarul Muzeului Bucovinei. Fascicula Stiintele Naturii, 12: 3-82. 